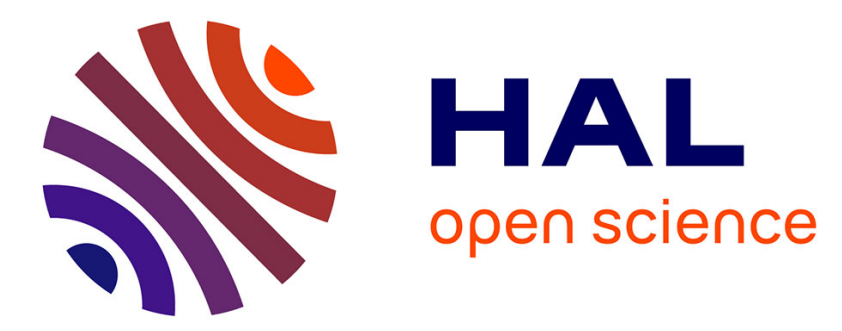

\title{
Experiments and numerical implementation of a boundary value problem involving a magnetorheological elastomer layer subjected to a non-uniform magnetic field
}

Charles Dorn, Laurence Bodelot, Kostas Danas

\section{To cite this version:}

Charles Dorn, Laurence Bodelot, Kostas Danas. Experiments and numerical implementation of a boundary value problem involving a magnetorheological elastomer layer subjected to a non-uniform magnetic field. Journal of Applied Mechanics, inPress, pp.1-12. 10.1115/1.4050534 . hal-03188506

\author{
HAL Id: hal-03188506 \\ https://hal-polytechnique.archives-ouvertes.fr/hal-03188506
}

Submitted on 2 Apr 2021

HAL is a multi-disciplinary open access archive for the deposit and dissemination of scientific research documents, whether they are published or not. The documents may come from teaching and research institutions in France or abroad, or from public or private research centers.
L'archive ouverte pluridisciplinaire HAL, est destinée au dépôt et à la diffusion de documents scientifiques de niveau recherche, publiés ou non, émanant des établissements d'enseignement et de recherche français ou étrangers, des laboratoires publics ou privés. 


\title{
Experiments and numerical implementation of a boundary value problem involving a magnetorheological elastomer layer subjected to a non-uniform magnetic field
}

\author{
Charles Dorn \\ Graduate Aerospace Laboratories \\ California Institute of Technology \\ Pasadena, California 91125, USA \\ Email: cdorn@caltech.edu
}

\author{
Laurence Bodelot* \\ Solid Mechanics Laboratory (LMS) \\ CNRS, Ecole Polytechnique \\ Institut Polytechnique de Paris \\ 91128 Palaiseau, France \\ Email: laurence.bodelot@polytechnique.edu
}

\author{
Kostas Danas \\ Solid Mechanics Laboratory (LMS) \\ CNRS, Ecole Polytechnique \\ Institut Polytechnique de Paris \\ 91128 Palaiseau, France \\ Email: konstantinos.danas@polytechnique.edu
}

This study investigates experimentally and numerically the response of a magnetorheological elastomer (MRE) layer placed atop an electromagnetic coil. The MRE layer is deflected upon application of a current in the coil, which creates highly non-uniform magnetic fields. Isotropic as well as transversely isotropic layers (i.e., containing chains of magnetic particles) are tested experimentally, and the isotropic layer exhibits the largest deflection. To enhance the energetic efficiency of the model device, an iron core is introduced inside the electromagnetic coil, thereby leading to an increase of the resulting magnetic field near the center of the $M R E$ layer. In parallel, the boundary value problem (BVP)including the MRE layer, the coil, the core (if present) and the surrounding air-is modeled numerically. For this, a magneto-mechanical, vector-potential-based variational formulation is implemented in a standard three-dimensional finite element model at finite strains. For the material description, a recently proposed analytical homogenizationguided model is used to analyze the MRE in the "coil-only" configuration. It is then employed to predict the response of the layer in the "coil plus core" configuration, thus circumventing the need for a separate material characterization procedure. The proposed numerical simulation strategy provides a deeper understanding of the underlying complexity of the magnetic fields and of their interaction with the MRE layer. This study also reveals the importance of modeling the entire setup for predicting the response of MRE materials and, as a result, constitutes a step towards designing more

\footnotetext{
*Address all correspondence to this author.
}

efficient MRE-based devices.

\section{Introduction}

Magnetorheological elastomers (MREs) are man-made smart composite materials obtained by dispersing magnetic particles in an elastomer matrix. Rigbi and Jilken [1] have been the first to conduct tests on MREs and thereby to introduce previously unknown magneto-mechanical effects in soft polymers. Indeed, the soft mechanical behavior of the matrix, combined with the magnetic properties of the particles, allow these flexible composites not only to alter their viscoelastic properties and stiffness but also to exhibit large displacements and/or deformations in response to external magnetic fields. While the former effect can be exploited in controllable-stiffness devices for numerous advanced damping applications [2] (ranging from automobile [3-5] to prostheses [6] to adaptive materials applications [7,8]), the latter effect is of interest for haptic devices, shape control or actuators [9-11].

MRE materials are thus undeniably appealing for modern high-tech applications. However, as pointed out in a recent review [11], they have been seldom utilized in commercial applications because the design of MRE-based devices has been so far devised only through a rather empirical approach. Previous studies presenting theoretical and numerical modeling of MRE [12-25] or h-MRE (i.e., with particles exhibiting remanent magnetization) [19, 20, 26,27] samples or structures have largely focused on magneto-mechanical 
(microstructure-based or continuum-based) material modeling and on uniformly applied magnetic far fields. Moreover, despite a potential for slower time response applications in actuators or shape control, only a handful of studies have been dedicated to characterizing experimentally the coupled magneto-mechanical behavior of MREs (or of MRE-based structures and devices) under low-rate loadings [19, 28-30]. Furthermore, in these experimental studies, the applied magnetic field has also been consistently uniform.

The present study builds upon works undertaken in order to characterize experimentally and theoretically the coupled magneto-mechanical behavior of MREs at large strains and large uniform magnetic fields. Specifically, a comprehensive experimental study [30] has revealed that characterization of the coupled magneto-mechanical behavior of MRE materials necessitated specific samples and setups. Additionally, shape effects and instabilities inherently present in MRE samples impose numerical parameter identification instead of straightforward analytical identification. Hence, in parallel, numerically-assessed, microstructural analytical, explicit models have been developed for MREs allowing for an efficient numerical implementation in finite element (FE) codes $[16,23,24]$. Here, experiments and theory are combined to build and study a complete but highly non-trivial MRE-based boundary value problem (BVP) with non-uniform magnetic fields at finite strains, where material identification is performed directly within the proposed device. In particular, a simple model device (akin to a haptic surface) is proposedconsisting of a magnetorheological layer resting atop an electromagnetic coil - and tested for different MRE layer parameters (with or without particle field-structuring) and configurations (presence or absence of an iron core in the electromagnetic coil). The MRE device is faithfully reproduced and numerically simulated with the finite element method. To model the device, a vector-potential-based, finite-strain, magneto-mechanical variational formulation is proposed and implemented in three-dimensions using standard finite elements with standard nodal connectivity. The simulations accurately capture the behavior of the device and produce reliable predictions of its behavior under a different configuration. Experiments serve as a benchmark for the numerical simulations, which in turn provide further insight into the magneto-mechanical coupling mechanisms, as well as into the non-uniformity of the applied magnetic fields at play.

\section{Experiments}

\subsection{Sample fabrication}

The MRE samples are made of a very soft and stretchable two-part silicone elastomer (Ecoflex 00-20 from Smooth-On Inc., USA) in which are dispersed soft (i.e., without remanence) iron particles having a $3.5 \mu \mathrm{m}$ median diameter (carbonyl iron powder (CIP) SM from BASF). For practical convenience during polymer fabrication, particle content is expressed in parts per hundred rubber (phr), which corresponds to the mass of particles per hundred parts of compounded elastomer mass. In this section, the corresponding volume fraction $c$ of particles (see [30] for details on the conversion) is mentioned in parenthesis for clarity since such a quantity is relevant in the modeling of the magnetomechanical couplings and is used as such in Sec. 3. Samples of $70 \mathrm{phr}(c=0.08)$ are considered here. After mixing the particles without prior chemical treatment $[30,31]$ in Part A of the silicone elastomer, Part B is added and the mix is further stirred thoroughly. The mix is then poured in a copper mold, shaped so as to yield an MRE disk $3 \mathrm{~mm}$ in thickness and $44 \mathrm{~mm}$ in diameter. The copper mold consists of heating plates so that curing of the samples $\left(1 \mathrm{~h}\right.$ at $\left.70^{\circ} \mathrm{C}\right)$ can either be performed outside or inside a magnetic field generated by a large electromagnet (more details on the fabrication procedure can be found in [30]). This leads, respectively, to either isotropic MRE samples or to transversely isotropic samples, also called "field-structured" samples, with particle chains aligned either within the plane of the disk (so-called "perpendicular" to the subsequently applied magnetic field) or along the thickness of the disk (so-called "parallel" to the subsequently applied magnetic field).

Overall, three types of $70 \mathrm{phr}(c=0.08)$ MRE disks are fabricated, denoted henceforth as isotropic, parallel and perpendicular.

\subsection{Setup}

In the model device considered in this study, the magnetic field is generated by an electromagnetic coil manufactured by the Caylar company (France) and powered by a $20 \mathrm{~V}-20 \mathrm{~A}$ source. The winding is made with a pair of insulated copper wires $1.2 \mathrm{~mm}$ in diameter and contains 360 turns. The coil has the following dimensions: $20 \mathrm{~mm}$ inner diameter, $65 \mathrm{~mm}$ outer diameter and $33 \mathrm{~mm}$ height. This coil is surrounded by a pure iron housing of $40 \mathrm{~mm}$ inner diameter and $90 \mathrm{~mm}$ outer diameter that offers extra space for $10^{\circ} \mathrm{C}$ water provided by a chiller to circulate and cool down the system. A tapered pure iron core can be installed at the center of the coil to enhance the intensity of the magnetic field at the location where the MRE layer is to be installed. Note that the top of the iron core protrudes out of the coil. This coil within its iron housing is inserted in a 3Dprinted plastic casing that permits placing and then clamping an MRE disk on top of the coil. The diameter of the MRE disk remaining outside of the clamp is then $30 \mathrm{~mm}$ and the height of the clamping part of the casing is adjustable. A photograph of the electromagnetic coil and its tapered pure iron core is provided in Fig. 1a, and a schematic of the complete device, including the plastic casing and the clamp, is shown in Fig. $1 \mathrm{~b}$.

Tests can be carried out either with or without the tapered iron core, and the MRE disk is then installed to maintain a vertical gap of $3 \mathrm{~mm}$ with either the top of the coil or the top of the iron core. When the iron core is not installed, the maximum current intensity applied to the electromagnetic coil is $20 \mathrm{~A}$ (restricted by the power source) whereas, when the iron core is installed, the maximum applied intensity is chosen to be limited at $2.7 \mathrm{~A}$. As a matter of fact, beyond this threshold, the layer is at risk of entering in contact with the core despite the spacer installed for elevating the 

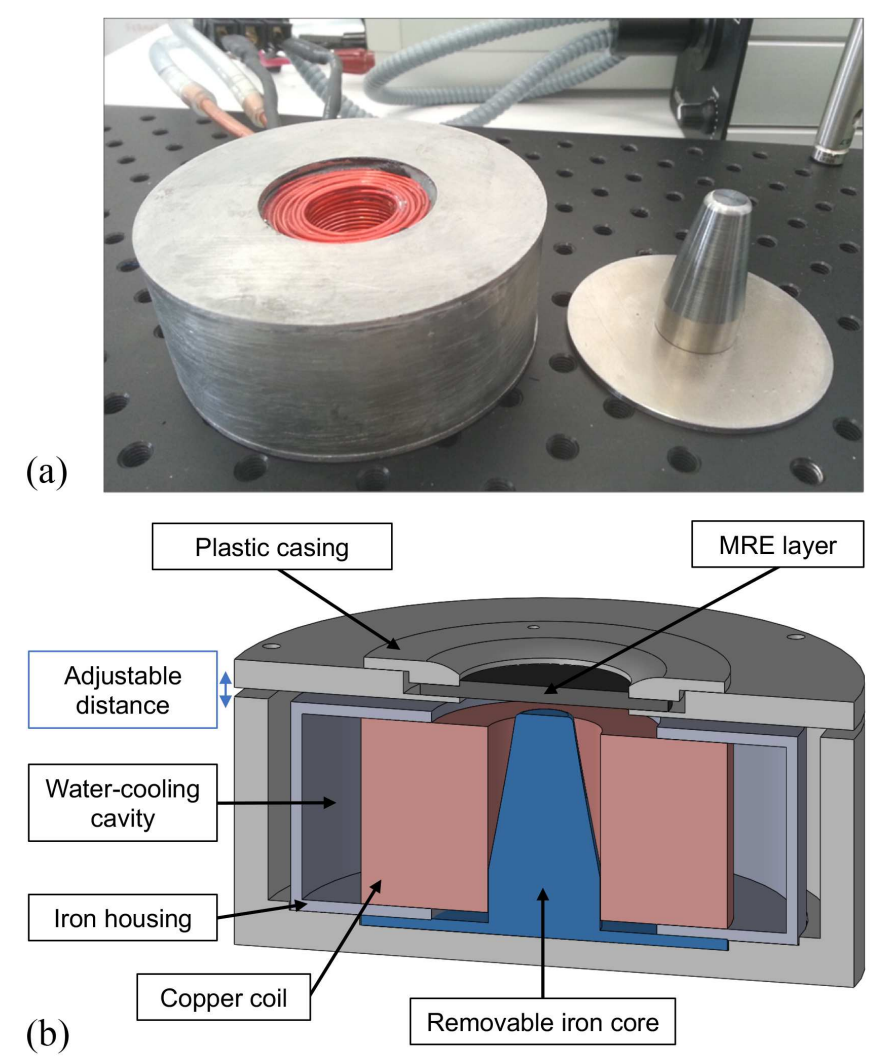

Fig. 1. (a) Photograph showing the coil surrounded by a pure iron housing (left) and the tapered iron core that can be placed at its center to enhance the generated magnetic field (right). (b) Schematic (cross-section view along a symmetry plane) of the complete device comprising an MRE layer placed atop the electromagnetic coil with the iron core, iron housing, plastic casing and clamp.

disk and maintaining a $3 \mathrm{~mm}$ gap. This attests to the efficacy of the core in enhancing the magnetic field intensity at a given current and thus in reducing the need for current to power MRE-based devices.

During the experiments, the displacements at the surface of each MRE layer are monitored in-situ by 3D Digital Image Correlation (3D-DIC) using the Correlated Solutions software. In accordance with the requirements of DIC [32], a random speckle pattern is applied onto the MRE layer using white spray paint (here, the sample itself constitutes a dark background). The MRE layer is lit symmetrically by two LED optical fibers to obtain proper contrast without reflections when the surface deforms. The deforming MRE layer is then imaged by two Pike 505-B cameras equipped with Tamron lenses and installed symmetrically on both sides of the prototype so as to form an angle with its normal (see Fig. 2). A calibration of the system with a normalized target permits the Correlated Solutions 3D-DIC software to locate the cameras in space and relate the displacements of the speckle pattern to the relative displacement of the layer in the three-dimensional space.

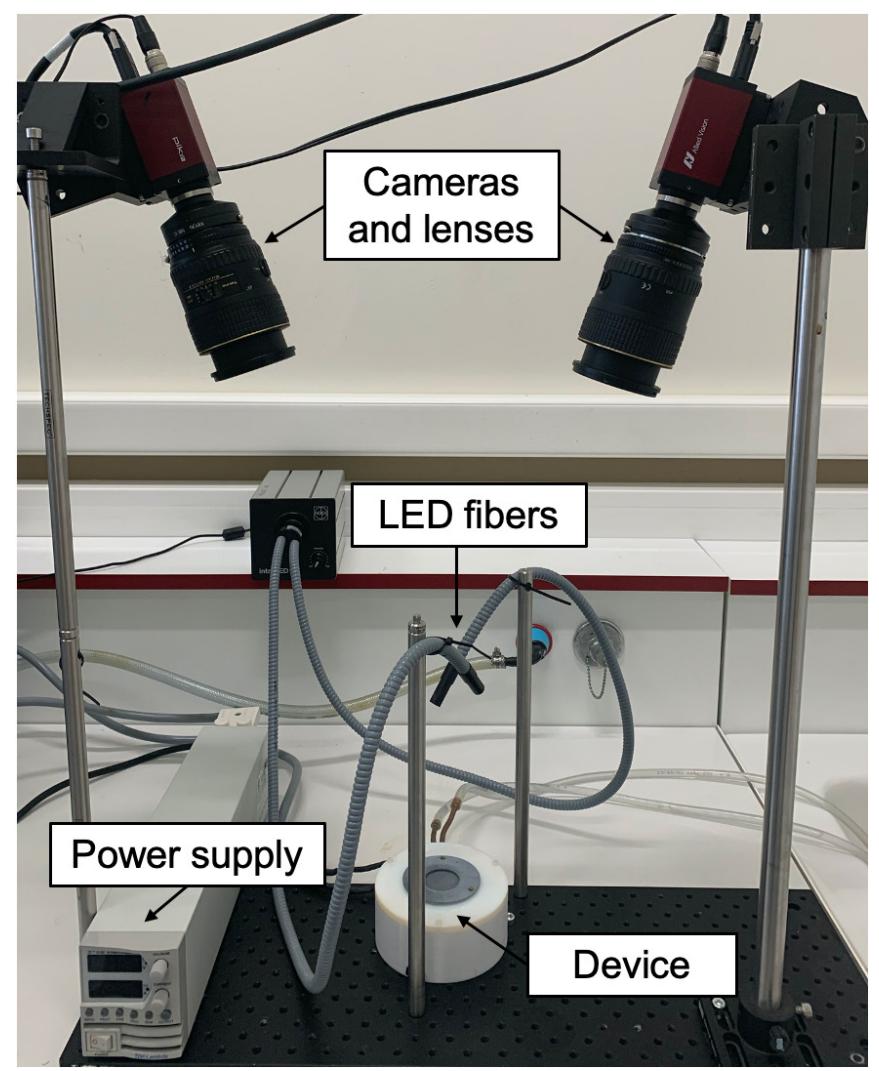

Fig. 2. 3D-DIC experimental setup used to access the displacements at the surface of the MRE layers under increasing magnetic field generated within the model device.

\subsection{Experimental results}

During each test, an increasing current intensity is applied to the electromagnetic coil, thus yielding an increasing magnetic field. Three tests per configuration are conducted and average values are reported along with error bars corresponding to a standard deviation. In what follows, the vertical position at the center of the upper surface of the disk is plotted as a function of the applied current intensity for each test case.

\subsubsection{Effect of particle field-structuring}

$70 \mathrm{phr}(c=0.08)$ MRE disk layers exhibiting particle field-structuring as described in Sec. 2.1 (i.e., isotropic, perpendicular and parallel to the field) are first tested in the device with no iron core. The vertical positions at the center of their upper surfaces are plotted in Fig. 3 as the applied current increases.

Considering that the main direction of the magnetic field is along the revolution axis of the coil, the results plotted in Fig. 3 show that the smallest deflection is obtained when particle chains are parallel to the applied field. The maximum deflection appears in the case of the isotropic MRE layer, while the MRE layer with the particle chains aligned perpendicular to the magnetic field show intermediate deflection.

These results shall be commented in light of those obtained in the context of the MRE material characterization 


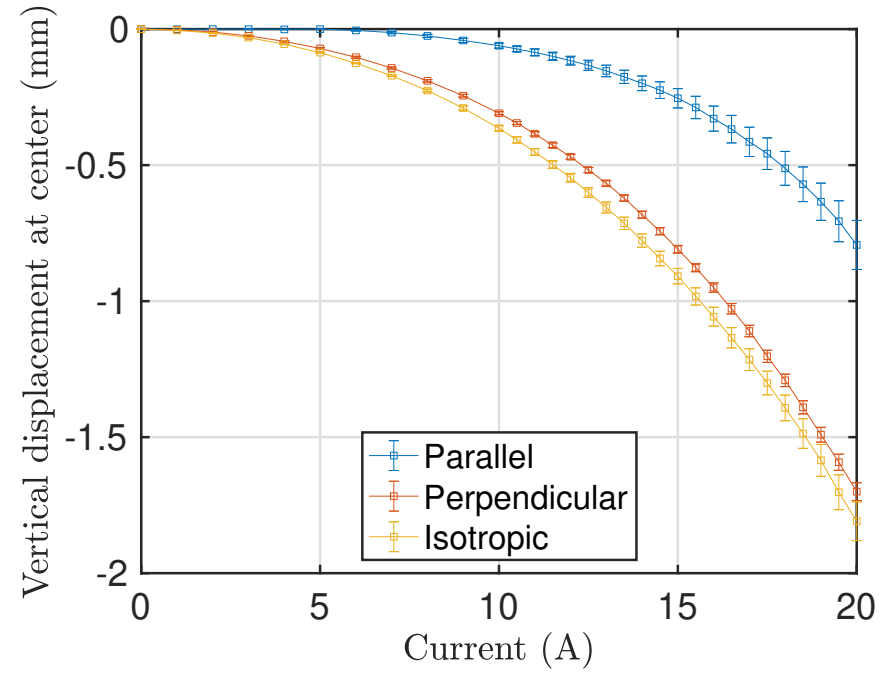

Fig. 3. Effect of particle field-structuring on the device behavior: vertical position at the center of the upper surface of the disk for $70 \mathrm{phr}(\mathrm{c}=0.08)$ disk layers that are isotropic, perpendicular and parallel. The reported values correspond to the average over three tests conducted in the same configuration and error bars represent a standard deviation.

conducted in [30] as well as of the instability results obtained in [29]. In the present device, the MRE sample is fairly slender, with its long dimension positioned perpendicular to the applied magnetic field. Hence, upon application of the magnetic field, this causes a strong attraction of the MRE sample. This is directly related to the well-known compass effect and to previously observed overall sample instabilities (constrained by boundary conditions), which occur in all sample configurations, i.e., isotropic, parallel and perpendicular. Furthermore, it was shown that particle chains have an affect akin to fibers in composite materials, thereby reinforcing the stiffness of the material along their direction [13,30,33]. Hence, the perpendicular configuration, even if known to be the most unstable [30,34], is also rather stiff mechanically due to the anisotropy induced by the particle chains. The same is also true for the parallel configuration, which despite having the largest magnetic susceptibility along the particle chains $[13,30,33]$, is simultaneously the stiffest mechanically among all configurations considered here. This stiffness is further enhanced by the presence of the magnetic field in this case, which tends to keep the particles parallel to the field. This interplay between the mechanical stiffness and magnetic properties leads to an optimal response obtained in the isotropic configuration. The latter is mechanically the softest of all, while any potential benefits of field-structuring in the parallel and perpendicular configurations are, in the present case, outweighed by the corresponding mechanical stiffening.

\subsubsection{Influence of the core}

In the following, focus is set on the optimal case of the isotropic MRE layer. It is further tested in the presence of the iron core, and the vertical position at the center of its upper

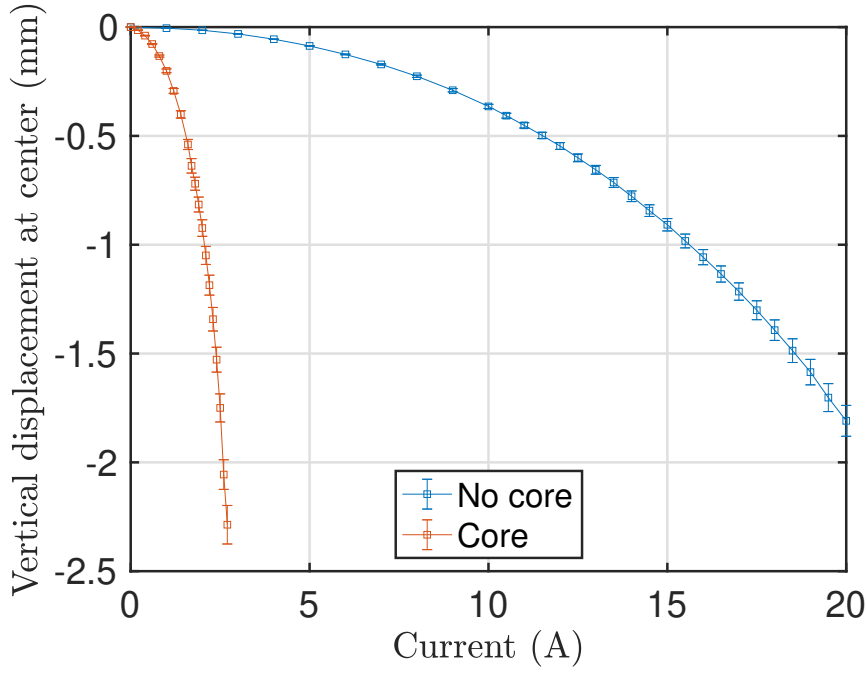

Fig. 4. Influence of the core on the device behavior: vertical position at the center of the upper surface of the disk for a $70 \mathrm{phr}(\mathrm{c}=0.08)$ isotropic disk layer in the presence of and in the absence of the core. The reported values correspond to the average over three tests conducted in the same configuration and error bars represent a standard deviation.

surface is plotted in Fig. 4.

As expected, Fig. 4 readily shows that installing an iron core within the coil has a strong effect on the deflection evolution of the layer. Indeed, the iron core provokes a magnetic field concentration at the center of the MRE disk layer, as will be highlighted in the numerical simulations presented in Sec. 4.2. In the absence of an iron core, the maximum displacement attainable is $-1.8 \mathrm{~mm}$ at $20 \mathrm{~A}$ whereas, with the iron core installed, a displacement of $-2.3 \mathrm{~mm}$ is obtained at the maximum applied current of $2.7 \mathrm{~A}$. To highlight the influence of the core, one can remark that a displacement of $-1.8 \mathrm{~mm}$ is reached at only about $2.5 \mathrm{~A}$ with the core, versus $20 \mathrm{~A}$ with no core. This clearly demonstrates the interest of the iron core for lowering the input current. Note that no optimization was conducted for the design of the iron core and this could be an interesting avenue to consider when power consumption is an important design parameter, or when power is supplied by standard batteries. This, nonetheless, requires the capacity to model such electromagnets and the non-uniform magnetic fields they generate, simultaneously with the resulting finite displacements and deformations of the soft components (e.g., the MRE layer in the present case).

\section{Theory and numerical implementation}

In this section, a general-purpose numerical framework capable of modeling the device is developed. To capture the full BVP, a vector potential-based magneto-mechanical variational principle is proposed that can describe the MRE layer, electromagnetic coil, iron core, and surrounding air, all with a single user-element definition. Modeling all components of the device creates a useful and realistic problem setting where the sole input is the current applied to the 
coil, matching the experimental setting. Furthermore, detailed non-uniform magnetic fields can be captured since the air surrounding the device is modeled. Based on this framework, a three-dimensional finite element is implemented in finite strain.

\subsection{A single variational principle}

First, a total Lagrangian variational formulation is established, from which a finite-strain, magneto-elastic, finite element framework is defined. More generally, consider a finite volume $V$ with boundary $\partial V$ occupied by a magneto-elastic material. A material point at position $\mathbf{X}$ in the undeformed configuration is mapped to $\mathbf{x}$ in the deformed configuration, via the displacement vector $\mathbf{u}$ as $\mathbf{x}=\mathbf{X}+\mathbf{u}(\mathbf{X})$. The deformation gradient is then given by $\mathbf{F}=\mathbf{I}+$ Gradu, where Grad denotes the gradient operator with respect to $\mathbf{X}$. In addition, the reference density of the solid $\rho_{0}$ is related to the current density $\rho$ by $\rho=J \rho_{0}$, where $J=\operatorname{det} \mathbf{F}>0$.

In order to parameterize the magnetic field, there is a choice as to which variable is considered as independent (see for instance equivalent formulations in [15, 23]). In this work, the magnetic field $\mathbf{B}$ is chosen as the independent magnetic variable, which, in turn, is described in terms of the magnetic vector potential $\mathbf{A}$ by $\mathbf{B}=\operatorname{CurlA}$ (where the "Curl" operator is defined with respect to the undeformed configuration). This is a convenient choice because on one hand, $\mathbf{A}$ is work-conjugate to the current density $\mathbf{J}$ (which leads to a straightforward means of modeling the coil) and on the other hand, it leads identically to DivB $=\mathbf{0}$ (where the "Div" operator is defined with respect to the undeformed configuration). In addition, one can show by standard pushforward operations that the Eulerian magnetic field is given by $\mathbf{b}=J^{-1} \mathbf{F B}$. Henceforth, unless stated otherwise, lower case variables represent Eulerian quantities, while upper case ones represent Lagrangian quantities. The conjugate stress and h-fields are defined later in this section.

Building on the works of [35] and [36], the total potential energy contained in $V$ is

$$
\begin{aligned}
\mathcal{P}(\mathbf{u}, \mathbf{A})= & \int_{V} W(\mathbf{F}(\mathbf{u}), \mathbf{B}(\mathbf{A})) \mathrm{d} V-\int_{V}(\mathbf{J} \cdot \mathbf{A}+\mathbf{f} \cdot \mathbf{u}) \mathrm{d} V \\
& -\int_{\partial V} \mathbf{T} \cdot \mathbf{u} \mathrm{d} S+\frac{1}{2 \mu_{0} \xi} \int_{V}(\operatorname{Div} \mathbf{A})^{2} \mathrm{~d} V,
\end{aligned}
$$

where $\mathbf{u}$ is a kinematically admissible displacement field in the usual sense and $\mathbf{A}$ is the vector potential to be discussed in the following, while the corresponding boundary conditions are detailed in Sec. 3.3.2. This formulation is based on the eddy current approximation, which consists of neglecting the electric displacement currents in the Maxwell equations [37]. Such an approximation is valid for low-rate quasi-static loading conditions such as those in the present study. The first term captures the internal energy of the material that occupies $V$. The energy density $W$ is defined for each material in the device in Sec. 3.2. The second and third terms represent external work due to body forces $\mathbf{f}$, applied traction $\mathbf{T}$, and current density $\mathbf{J}$. Inclusion of the $\mathbf{J} \cdot \mathbf{A}$ term is consistent with the source term in Ampère's law [36]. By incorporating the work done by the current density in the variational formulation, electromagnets can conveniently be modeled by assuming a continuum current density.

The last term in (1) serves to introduce the so-called Coulomb gauge constraint $\operatorname{DivA}=\mathbf{0}$. As mentioned previously, only the rotational part (i.e., Curl) of $\mathbf{A}$ affects the magnetic field $\mathbf{B}$, and as a result its divergence remains undetermined. To resolve this indeterminacy, several approaches have been proposed in the literature of electro-magnetics using the so-called edge elements (see for instance [38] and numerous works resulting from that study). Nonetheless, such a formulation expresses the vector-potential as an unknown at the edges of the element and not the nodes. In turn, the continuity of the displacement field in a finite-strain mechanical problem with multiple (perfect) interfaces requires the displacement to be defined at the nodes. This observation makes the combination of these two formulations extremely cumbersome technically. Consequently, Coulomb [39] and several other authors thereafter [39-43] have proposed the use of the last term in (1) to guarantee the uniqueness of the vector potential as well as its "continuity" across material interfaces (such as material/air/coil), without affecting in essence the primary magnetic field $\mathbf{B}$. The advantage of such a formulation allows the present form of a standard displacement-based finite element formulation to be maintained, and leads to a straightforward implementation. Note further that the constraint DivA $=\mathbf{0}$ may be implemented with different methods. For simplicity, in the present work, the Coulomb gauge is dealt with via the penalty method with $\xi$ denoting the penalty parameter that should take small values for the constraint to be satisfied up to a numerical tolerance. The permeability of vacuum $\mu_{0}$ is introduced simply for dimensional reasons. This term does not have physical meaning other than to enforce uniqueness of $\mathbf{A}$. In practice, the exact value of the penalty $\xi$ does not affect numerical solutions so long as it is sufficiently small and the corresponding term is under-integrated since it behaves as a strong constraint. In this study, a value $\xi=10^{-6}$ delivers converged and stable numerical results.

Then, minimization of $\mathcal{P}$ with respect to $\mathbf{u}$ and $\mathbf{A}$, implies setting the first variation of $\mathcal{P}$ equal to zero, i.e.,

$$
\begin{aligned}
\delta \mathcal{P}= & \int_{V}\left(\frac{\partial W(\mathbf{F}, \mathbf{B})}{\partial \mathbf{F}} \cdot \delta \mathbf{F}+\frac{\partial W(\mathbf{F}, \mathbf{B})}{\partial \mathbf{B}} \cdot \delta \mathbf{B}\right) \mathrm{d} V \\
& -\int_{V}(\mathbf{J} \cdot \delta \mathbf{A}+\mathbf{f} \cdot \delta \mathbf{u}) d V-\int_{\partial V}(\mathbf{T} \cdot \delta \mathbf{u}) \mathrm{d} S \\
& +\frac{1}{\mu_{0} \xi} \int_{V} \operatorname{Div} \mathbf{A} \cdot \operatorname{Div} \delta \mathbf{A} \mathrm{d} V=0,
\end{aligned}
$$

where $\delta \mathbf{F}=\operatorname{Grad} \delta \mathbf{u}$ and $\delta \mathbf{B}=\operatorname{Curl} \delta \mathbf{A}$. From this expression, the first Piola-Kirchhoff stress and Lagrangian $h$-field are readily identified as

$$
\mathbf{S}=\frac{\partial W(\mathbf{F}, \mathbf{B})}{\partial \mathbf{F}}, \quad \mathbf{H}=\frac{\partial W(\mathbf{F}, \mathbf{B})}{\partial \mathbf{B}} .
$$


The Eulerian counterparts of these fields are obtained by standard push-forward operations [44], such that $\boldsymbol{\sigma}=$ $J^{-1} \mathbf{S F}^{T}$ and $\mathbf{h}=\mathbf{H F}^{-T}$.

Expression (2) constitutes the weak form of the mechanical equilibrium equations and Maxwell equations of magneto-statics (see detailed discussion in [45] for instance). Subsequently, the second variation of the total potential energy reads

$$
\begin{aligned}
\Delta \delta \mathcal{P}= & \int_{V}\left(\Delta \mathbf{F} \cdot \frac{\partial^{2} W}{\partial \mathbf{F} \partial \mathbf{F}} \cdot \delta \mathbf{F}+\Delta \mathbf{F} \cdot \frac{\partial^{2} W}{\partial \mathbf{F} \partial \mathbf{B}} \cdot \delta \mathbf{B}\right. \\
& \left.+\Delta \mathbf{B} \cdot \frac{\partial^{2} W}{\partial \mathbf{B} \partial \mathbf{F}} \cdot \delta \mathbf{F}+\Delta \mathbf{B} \cdot \frac{\partial^{2} W}{\partial \mathbf{B} \partial \mathbf{B}} \cdot \delta \mathbf{B}\right) \mathrm{d} V \\
& +\frac{1}{\mu_{0} \xi} \int_{V} \operatorname{Div} \Delta \mathbf{A} \cdot \operatorname{Div} \delta \mathbf{A} \mathrm{d} V .
\end{aligned}
$$

Use of expressions (2) and (4) allows for a standard finite element discretization and resolution by standard NewtonRaphson schemes (see for instance details in [41]). In the present example, a three-dimensional finite strain magnetoelastic user-element is implemented in ABAQUS.

\subsection{Constitutive models}

Each component of the boundary value problem at hand is modeled by use of a suitable constitutive model defined for each material. Following recent work [21,23], the energy density per unit referential volume of a magneto-elastic material can be partitioned into contributions from mechanical, magnetic, and vacuum terms, i.e.,

$$
\begin{aligned}
W(\mathbf{F}, \mathbf{B}) & =\Psi^{\operatorname{mech}}\left(I_{1}, J\right)+\Psi^{\operatorname{mag}}\left(J, I_{5}\right) \\
& +\Psi^{\operatorname{vac}}\left(J, I_{5}\right)
\end{aligned}
$$

The corresponding magneto-mechanical invariants are defined as

$$
I_{1}=\mathbf{F}: \mathbf{F}, \quad J=\operatorname{det} \mathbf{F}, \quad I_{5}=\mathbf{F B} \cdot \mathbf{F B}
$$

For simplicity in the numerical implementation, a quasiincompressible form of the models is used. That is also in accord with experiments [30] that show a quasi-incompressible response of the present MREs (i.e. $J \approx 1$ but not equal to unity).

Each of the four materials in the device (MRE, iron, air, and coil) are modeled with the same energy density defined in equation (5), but with different material coefficients. Since the core and coil are modeled as rigid and the air is modeled with negligible stiffness, the mechanical contribution $\Psi^{\text {mech }}$ is most relevant for the MRE. Consequently, the mechanical energy density is chosen to model the MRE and is taken from the family of models proposed in [46]. The polymeric matrix phase is modeled as a Neo-Hookean material and the iron particles as mechanically rigid. The corresponding continuum (homogenized) energy density for the MRE reads

$$
\begin{aligned}
\Psi^{\text {mech }}\left(I_{1}, J\right)= & \frac{G_{\mathrm{m}}}{2(1-c)^{5 / 2}}\left[I_{1}-3-2 \ln J\right] \\
& +\frac{G_{\mathrm{m}}^{\prime}}{2(1-c)^{6}}(J-1)^{2}
\end{aligned}
$$

where $G_{\mathrm{m}}$ and $G_{\mathrm{m}}^{\prime}$ are the Lamé moduli of the polymer matrix phase and $c$ denotes the volume fraction of the particles. Note that the magneto-elastic response depends on the particle content expressed as a volume fraction $c$ and not as phr. Nevertheless, one can obtain the first from the second by using the density of the matrix $\rho_{\mathrm{m}}=1,070 \mathrm{~kg} / \mathrm{m}^{3}$ and the particles $\rho_{\mathrm{p}}=7,874 \mathrm{~kg} / \mathrm{m}^{3}$ [30]. Table 1 summarizes the moduli used to model each material. To model the quasiincompressible matrix of the MRE, the bulk modulus is taken as $G_{\mathrm{m}}^{\prime}=200 G_{\mathrm{m}}$. The shear modulus of the matrix is fit as $G_{\mathrm{m}}=0.0075 \mathrm{MPa}$ based on the experiment without the iron core, which is discussed in Sec. 4.1. The coil and core are modeled as nearly rigid by taking sufficiently large values for the moduli in (7).

Ideally, the air should have zero stiffness. In this modeling framework, however, a very soft material description for the air is employed, following existing literature [17]. That requires small, but nonetheless finite values for the mechanical air properties. As a consequence, the stiffness of the air affects the solution as a whole, but only weakly. This effect only leads to the use of slightly larger material properties (e.g., magnetic susceptibility and shear modulus) for the MRE layer for a good calibration of the model. Such a deviation from the actual properties is on the order of a couple of percents. Alternative ways to deal with the air [21] may improve on this aspect, however, the difficulty in the present problem lies in the very complex air geometry that results from the device itself. Thus, the simple but fairly efficient approach of a very soft mechanical energy has been used for the air.

In turn, the purely magnetic contribution is taken as

$$
\begin{aligned}
\Psi^{\operatorname{mag}}\left(J, I_{5}\right) & =-\frac{\chi}{2 \mu_{0}(1+\chi)} \frac{I_{5}}{J^{2}} 2 \mathcal{F}_{1}\left[\frac{1}{k}, \frac{2}{k}, 1+\frac{2}{k},\right. \\
& \left.-\left(\frac{\chi}{\mu_{0}(1+\chi) m^{s}} \frac{\sqrt{I_{5}}}{J}\right)^{k}\right],
\end{aligned}
$$

where $m^{s}$ and $\chi$ are the magnetic saturation and magnetic susceptibility of the MRE, while ${ }_{2} \mathcal{F}_{1}$ is the hypergeometric function. The hypergeometric function can be used to recover a wide range of magnetic responses from Langevin to hyperbolic tangent. For the MRE, a value $k=6$ defines accurately the magnetization curve, as discussed in [23]. The saturation and susceptibility of the MRE can be derived from 
Table 1. Material properties.

\begin{tabular}{lllll}
\hline Property & MRE & Iron & Coil & Air \\
\hline$G_{\mathrm{m}}(\mathrm{MPa})$ & 0.0075 & 0.75 & 0.75 & $3.75 \times 10^{-5}$ \\
$G_{\mathrm{m}}^{\prime}(\mathrm{MPa})$ & 1.5 & 3 & 3 & $7.5 \times 10^{-5}$ \\
$\mu_{0} m_{s}(\mathrm{~T})$ & - & 2.5 & 0 & 0 \\
$\chi$ & 0.235 & 2000 & 0 & 0 \\
$c$ & 0.08 & - & - & - \\
$\mu_{0} m_{\mathrm{p}}^{s}(\mathrm{~T})$ & 2.5 & - & - & - \\
$\chi_{\mathrm{p}}$ & 30 & - & - & - \\
\hline
\end{tabular}

the matrix and particle constituents as [23]

$$
m^{s}=c m_{\mathrm{p}}^{s}, \quad \chi=\frac{3 c \chi_{\mathrm{p}}}{(2+c)+(1-c)\left(1+\chi_{\mathrm{p}}\right)},
$$

where the particles have volume fraction $c$, saturation magnetization $m_{\mathrm{p}}^{s}$ and magnetic susceptibility $\chi_{\mathrm{p}}$. The hypergeometric function is also suitable to model the magnetic response of iron, for which $k=2$ is used. Finally, the coil and air are not magnetic. Table 1 summarizes the magnetic parameters used for each material. Specifically, a very large susceptibility is used for the iron taken from standard handbooks. is

Finally, the contribution of the magnetic field in vacuum

$$
\Psi^{\operatorname{vac}}\left(J, I_{5}\right)=\frac{I_{5}}{2 \mu_{0} J}
$$

This term is present in all materials and serves to describe the presence of the background magnetic field [35].

It is remarked here that a more elaborate constitutive law could also include a coupling term $\Psi^{\text {couple }}$ in equation (5) to describe magnetostrictive coupling in the MRE. For this specific boundary value problem, however, the coupling term has been shown (not included here for brevity) to have negligible contribution to the simulations (see also [21]), due to the fairly small strains (but significant rotations and thus the finite strain framework) in the MRE disk. Thus, the coupled term in the constitutive equation is omitted and the reader is referred to [23] for a more detailed discussion of this point.

\subsection{Numerical implementation}

A finite element model is implemented in ABAQUS via a user-element routine to represent the device. The model describes as faithfully as possible the three-dimensional boundary value problem of the device, with the sole input being the current applied to the electromagnetic coil. To capture this problem setting, the volume containing the MRE layer, coil, iron core and casing, and surrounding air must be modeled.

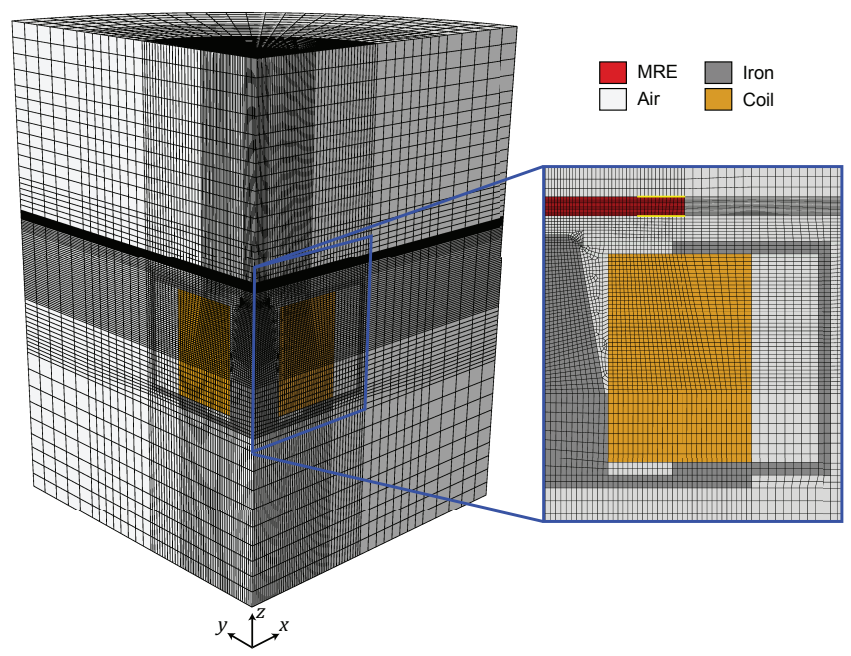

Fig. 5. Finite element mesh for the device configuration with the core present.

The various aspects of the implementation are discussed in this section.

\subsubsection{Meshing}

A user element, based on the variational principle of Sec. 3.1 and constitutive model of Sec. 3.2, is used to model all materials in the problem. The user element is implemented as an 8-node hexahedral element with a $2 \times 2$ Gauss integration rule (except for the Coulomb gauge term that uses a single Gauss point in the middle of the element). There are six degrees of freedom per node describing the displacements $u_{i}(i=1,2,3)$ and magnetic vector potential $A_{i}(i=1,2,3)$. For elements belonging to the MRE phase, which is quasi-incompressible, reduced integration is also used for the term $(J-1)^{2}$.

By employing quarter-symmetry, a quarter-cylindrical domain is used to encompass the device and surrounding air. For resolving accurately the magnetic field, convergence is considered with respect to the domain size and use of Neumann and Dirichlet type boundary conditions for the vector potential (not shown here for brevity). A domain with a radius of $112 \mathrm{~mm}$ and a height of $161 \mathrm{~mm}$ is found to be sufficiently large, thereby leading to no differences in the MRE response irrespective of the type of far boundary conditions on the vector potential. As a consequence, further extending the domain has negligible effects on the solution. Fig. 5 shows the mesh, with each material highlighted. The device configuration with the core present is depicted in this figure. For the configuration with the core inserted, a mesh with 181,576 elements and 192,105 nodes is used. For the device configuration without the core, a mesh with 160,646 elements and 171,000 nodes is used. In both cases, the total size of the problem is on the order of $10^{6}$ degrees of freedom (as there are 6 degrees of freedom per node).

The mesh represents all components of the experimental setup shown in Fig. 1b, except the plastic casing. Since the plastic casing is non-magnetic, it exhibits negligible defor- 
mation, and does not interfere with the important parts of the device (e.g. MRE, core, coil). For this reason, it is replaced with undeformable air in the numerical model. To model the clamping of the MRE by the plastic casing, displacement boundary conditions are applied along the outer edge of the MRE surface, which is highlighted in yellow in Fig. 5.

\subsubsection{Boundary Conditions}

Three sets of boundary conditions are applied to the domain. The first set enforces symmetry along the $x z$ and $y z$ planes. The displacement degrees of freedom of nodes on these faces are constrained such that no displacement can occur normal to the plane of symmetry. To enforce symmetry of the magnetic field, the corresponding boundary conditions on the vector potential read

$$
\begin{aligned}
& A_{1}(y=0)=0,\left.\frac{\partial A_{2}}{\partial y}\right|_{y=0}=0, A_{1}(y=0)=0 \\
& \left.\frac{\partial A_{1}}{\partial x}\right|_{x=0}=0, A_{2}(x=0)=0, A_{3}(x=0)=0 .
\end{aligned}
$$

These conditions are consistent with tangential continuity $[42,43]$ of $\mathbf{A}$ across the symmetry planes (i.e., $\llbracket \mathbf{A} \rrbracket \times \mathbf{n}=\mathbf{0}$ with $\mathbf{n}$ denoting the normal to the symmetry plane).

The second set of boundary conditions is applied to the far-field boundary, which includes the top, bottom, and outer radial surface of the quarter-cylindrical domain. On the farfield boundary, either homogeneous Neumann or Dirichlet conditions can be enforced on all vector potential components. In this study, a sufficiently large domain is employed. Thus the obtained solutions are independent of the far boundary conditions on the vector potential.

The third set of boundary conditions models the clamping of the MRE by the plastic casing. Along the lower yellow segment on the MRE in Fig. 5, the z-displacement is fixed. Along the upper yellow segment, a vertical displacement of $-1 \mathrm{~mm}$ is applied following the experimental setup. Sliding is prevented by fixing all displacement components on the inner radius of the lower yellow segment. In reality, the clamping of the MRE by the casing may exhibit a more complex behavior. As the device is loaded and the MRE is pulled downward by the magnetic field, the grip of the casing may not be perfect and the MRE may slide with respect to the casing. A more complex model taking into account approximately the friction (see for instance [21]) could perhaps capture more accurately the clamping boundary condition. Nonetheless, the focus of this study is to capture the overall behavior of the device BVP and this simplified boundary condition will prove sufficient.

\subsubsection{Loading}

The source of loading in the device is the current applied to the electromagnetic coil. There is a trade-off regarding the level of detail of the model: more details can always be added to the model to better capture the experimental setting at the cost of working with an overly complex model. Instead of modeling individual loops of wire in the coil, a continuum model is adopted. The continuum model assumes axisymmetry and uniform current density, which provides a reasonable approximation for a thick coil with many turns [47]. Current density in the coil is approximated by

$$
\mathbf{J}=\mathbf{J}_{\Theta} \mathbf{e}_{\Theta}, \quad \mathbf{J}_{\Theta}=\frac{N I}{h\left(r_{o}-r_{i}\right)} .
$$

In this expression, $\mathrm{J}_{\Theta}$ is the current density along the angular direction $\mathbf{e}_{\Theta}$ of a cylindrical coordinate system describing the coil. The current in each of the $N$ turns of the wire is $I$ and $r_{i}=10 \mathrm{~mm}, r_{o}=32.5 \mathrm{~mm}$, and $h=33 \mathrm{~mm}$ are the inside radius, outside radius, and height of the coil, respectively.

To better capture the experimental setting, self-weight of the MRE is also considered, which is implemented as a body force $\mathbf{f}=-\rho g \mathbf{e}_{3}$ in the $z$ direction where $\rho=(1-c) \rho_{\mathrm{m}}+c \rho_{\mathrm{p}}$ and $g$ is acceleration due to gravity. Loading of the boundary value problem is performed as a three-step process. First, the clamp boundary condition is applied, representing the mounting of the MRE in the plastic casing. The second step is to apply gravitational loading. Finally, the current $I$ is applied to the coil, producing a magnetic field that loads the MRE.

\section{Discussion}

\subsection{Comparison between experimental and numerical results}

Figure 6 compares experimental and numerical vertical displacements at the center of the top surface of the isotropic MRE layer, with and without the core present. The constitutive model requires the calibration of the shear modulus of the MRE matrix $G_{\mathrm{m}}$ only, while the rest is taken from handbooks and earlier studies as reported in Table 1. To identify the value of $G_{\mathrm{m}}$, the experimental data from the "coil only" configuration is used to obtain a fit. A shear modulus of $G_{\mathrm{m}}=7.5 \mathrm{kPa}$ provides good agreement between the experiment and simulation for the "coil only" configuration. This value is in close agreement with earlier work using similar MRE materials [21,30].

The model additionally demonstrates predictive capability, which is evident through the good agreement between the experiment and simulations for the "coil plus core" configuration. This agreement is rather impressive given that the magnetic fields in the configurations with and without the core are very different, as discussed in Sec. 4.2. It is noted that no further calibration is performed to obtain the response with the core.

Specifically, the simulations are in excellent agreement with the experiments for a large range of applied current. As the MRE displacement increases, there is some divergence between the experimental and numerical curves (see Fig. 6). This is due to the complex boundary conditions of the plastic casing gripping the MRE. In the experiment, the MRE is not perfectly gripped by the casing and some sliding is inevitable. This sliding is not modeled in the present simu- 


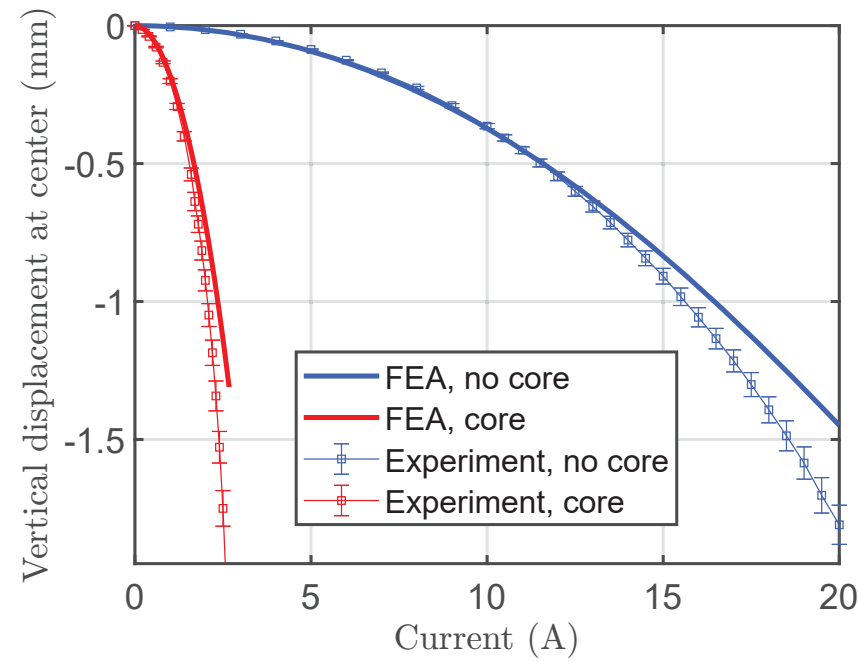

Fig. 6. Comparison between experiments and finite element simulations for device configurations with and without the core: vertical position at the center of the upper surface of the isotropic MRE layer.

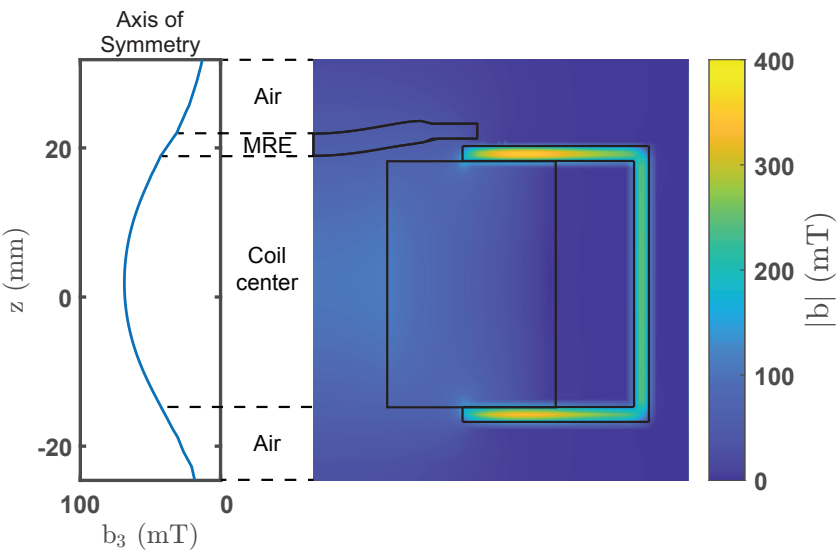

Fig. 7. Eulerian magnetic field $b$ plotted on the $x z$ face and along the axis of symmetry. The field corresponds to an applied current $\mathrm{I}=20 \mathrm{~A}$ in the "coil only" configuration.

lation, thus leading to a slightly stiffer response as the MRE disk displaces.

\subsection{Magnetic fields}

The simulations are now used to provide further insight into aspects of the device that are out of reach experimentally, such as a detailed description of the induced magnetic fields. Figs. 7 and 8 show the Eulerian magnetic fields $\mathbf{b}$ and h on the $x z$ face of the mesh (refer to Fig. 5) in the "coil only" configuration corresponding to a $20 \mathrm{~A}$ applied current. Similarly, Figs. 9 and 10 show the Eulerian magnetic fields on the $x z$ face in the "coil plus core" configuration corresponding to a $2.7 \mathrm{~A}$ applied current.

The simulated magnetic fields highlight the role of the core. By focusing but also intensifying the magnetic field near the MRE, the core leads to stronger attractive magnetic forces towards the MRE disk and thus to larger deflections at much lower applied currents. In contrast, the magnetic fields

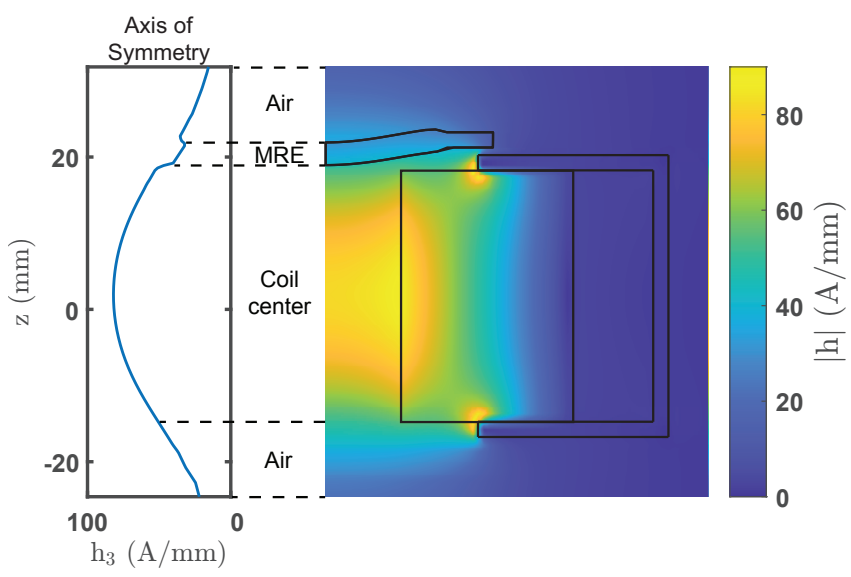

Fig. 8. Eulerian $h$-field plotted on the $x z$ face and along the axis of symmetry. The field corresponds to an applied current $\mathrm{I}=20 \mathrm{~A}$ in the "coil only" configuration.

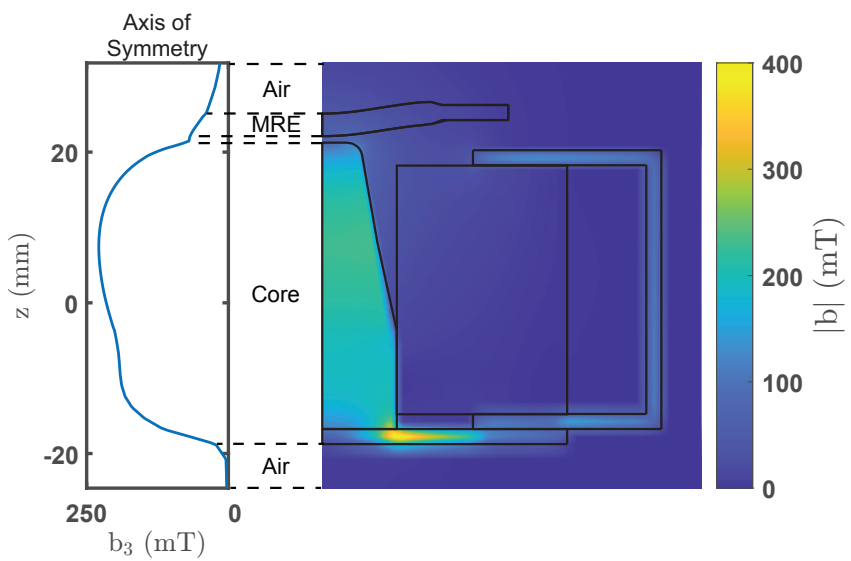

Fig. 9. Eulerian magnetic field $b$ plotted on the $x z$ face and along the axis of symmetry. The field corresponds to an applied current $\mathrm{I}=2.7 \mathrm{~A}$ in the "coil plus core" configuration.

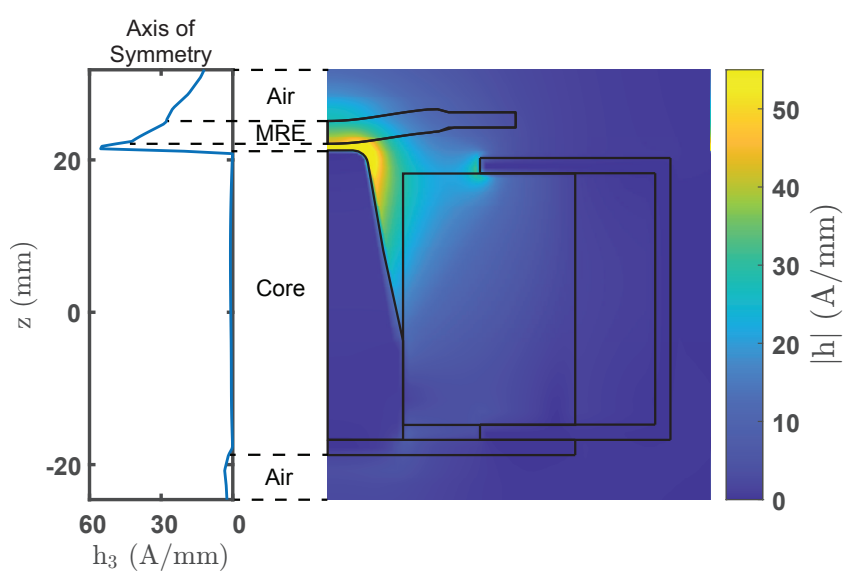

Fig. 10. Eulerian $h$-field plotted on the $x z$ face and along the axis of symmetry. The field corresponds to an applied current $\mathrm{I}=2.7 \mathrm{~A}$ in the "coil plus core" configuration. 
created by the coil in the "coil only" configuration strongly diminish near the MRE layer. Additionally, the fields are able to show the effect of surrounding materials, such as the iron casing (see, for instance, Figs. 7 and 8) as well as the effect of the iron core geometry which exhibits a rather non-uniform internal distribution of the magnetic field $b$ (see Fig. 9).

From a numerical standpoint, Figs. 7-10 demonstrate that highly non-uniform magnetic fields can be modeled by the presented formulation. Furthermore, jumps in the magnetic field at interfaces are properly captured with the proposed formulation. This is evident in the $h$-fields, where there are jumps on the top and bottom of the MRE layer as expected by the tangential-only continuity required for those fields.

\section{Conclusion}

In this work, experiments and numerical simulations of a model device composed of an electromagnetic coil that deflects a magnetorheological elastomer layer are presented. Experimental results show that particle field-structuring and their associated instabilities do not present any advantage in the proposed configuration. The fact that an isotropic MRE performs best is counter-intuitive considering the results from material characterization (see [30]), but is useful from a fabrication standpoint. Furthermore, the experiments show that the presence of an iron core inside the coil concentrates the magnetic field near the layer to cause large deflections at smaller applied currents.

Alongside the experiments, a general framework for numerical simulations of magneto-mechanical BVPs involving coils is presented. A magnetic vector potential variational framework is proposed, from which a three-dimensional magneto-elastic finite element model is derived at finite strains and arbitrary magnetic fields. The numerical implementation introduces two key features that contribute to its versatility. First, the external work due to current density is included in a Lagrangian formulation, allowing electromagnets to be modeled using an eddy current approximation. Second, a Coulomb gauge is enforced using a penalty term, ensuring uniqueness of the vector potential. Together, these terms allow for highly non-uniform magnetic fields generated by electromagnets to be captured along with a finite strain Lagrangian formulation.

The numerical implementation allows for versatile modeling of non-trivial magneto-mechanical BVPs since everything, from electromagnets to MREs to magnetic metals, can be modeled with a single user-element definition. Thus, simulations of the MRE device could be performed in a setting that parallels the experiment, where the only controlled variable is the current that is applied to the electromagnet. The model shows very good agreement with experiments in both of the considered device configurations, i.e., with and without the iron core. Here, it is important to recall that constitutive calibration is only performed in the "coil only" configuration, thereby circumventing the need for a separate material characterization procedure. In view of this, the model shows powerful predictive capability through agreement with experiment in the "coil plus core" configuration. Furthermore, the simulations provide insights that cannot be obtained experimentally, such as detailed magnetic fields, which properly reproduce jumps at material interfaces. Overall, the proposed simulation framework proves to be an accurate and robust tool for modeling complex BVPs involving MREs and, thus, constitutes a step towards designing more efficient MRE-based devices.

\section{Acknowledgements}

The authors acknowledge Tobias Pössinger for designing the coil used in the study and constructive discussions with Prof. N. Triantafyllidis. K.D. acknowledges the support from the European Research Council (ERC) under the European Union's Horizon 2020 research and innovation program (grant agreement No 636903 - MAGNETO). The computational part of this work was supported by the ANR, France under contract number ANR-10-EQPX-37. The work of C.D. was initially supported by the Ecole Polytechnique and its Foundation during AY 2017-2018 through the dual MS program with Caltech and subsequently by the Solid Mechanics Laboratory (LMS) of the Ecole.

\section{References}

[1] Rigbi, Z., and Jilken, L., 1983. "The response of an elastomer filled with soft ferrite to mechanical and magnetic influences". Journal of Magnetism and Magnetic Materials, 37(3), pp. 267-276.

[2] Li, Y., Li, J., Li, W., and Du, H., 2014. "A state-of-theart review on magnetorheological elastomer devices". Smart Materials and Structures, 23(12), p. 123001.

[3] Elie, L. D., Ginder, J. M., Nichols, M. E., and Stewart, W. M., 2002. "Variable stiffness bushing using magnetorheological elastomers". European Patent, EP0784163A.

[4] Crist, R. J., 2009. “Active vibrational damper". US Patent, US7584685B.

[5] Marur, P. R., 2013. "Magneto-rheological elastomerbased vehicle suspension”. US Patent, US20130087.

[6] Thorsteinsson, F., Gudmundsson, I., and Lecomte, C., 2015. "Prosthetic and orthotic devices having magnetorheological elastomer spring with controllable stiffness". US Patent, US9078734B.

[7] Ginder, J. M., Nichols, M. E., Elie, L. D., and Clark, S. M., 2000. "Controllable-stiffness components based on magnetorheological elastomers". In SPIE's 7th Annual International Symposium on Smart Structures and Materials, International Society for Optics and Photonics, pp. 418-425.

[8] Farshad, M., and Le Roux, M., 2004. "A new active noise abatement barrier system". Polymer testing, 23(7), pp. 855-860.

[9] Böse, H., Rabindranath, R., and Ehrlich, J., 2012. "Soft magnetorheological elastomers as new actuators for 
valves". Journal of Intelligent Material Systems and Structures, 23(9), pp. 989-994.

[10] Stepanov, G., Kramarenko, E. Y., and Semerenko, D., 2013. "Magnetodeformational effect of the magnetoactive elastomer and its possible applications". In J. Phys.: Conf. Ser, Vol. 412, p. 012031.

[11] Ahamed, R., Choi, S.-B., and Ferdaus, M. M., 2018. "A state of art on magneto-rheological materials and their potential applications". Journal of Intelligent Material Systems and Structures, 29(10), pp. 2051-2095.

[12] Borcea, L., and Bruno, O., 2001. "On the magnetoelastic properties of elastomer-ferromagnet composites". Journal of the Mechanics and Physics of Solids, 49(12), pp. 2877-2919.

[13] Danas, K., Kankanala, S., and Triantafyllidis, N., 2012. "Experiments and modeling of iron-particle-filled magnetorheological elastomers". Journal of the Mechanics and Physics of Solids, 60(1), pp. 120 - 138.

[14] Kalina, K. A., Metsch, P., and Kästner, M., 2016. "Microscale modeling and simulation of magnetorheological elastomers at finite strains: A study on the influence of mechanical preloads". International Journal of Solids and Structures, 102-103, pp. 286-296.

[15] Danas, K., 2017. "Effective response of classical, auxetic and chiral magnetoelastic materials by use of a new variational principle". Journal of the Mechanics and Physics of Solids, 105, pp. 25-53.

[16] Lefèvre, V., Danas, K., and Lopez-Pamies, O., 2017. "A general result for the magnetoelastic response of isotropic suspensions of iron and ferrofluid particles in rubber, with applications to spherical and cylindrical specimens". Journal of the Mechanics and Physics of Solids, 107, pp. 343-364.

[17] Keip, M.-A., and Rambausek, M., 2017. "Computational and analytical investigations of shape effects in the experimental characterization of magnetorheological elastomers". International Journal of Solids and Structures, 121, pp. 1-20.

[18] Kalina, K. A., Brummund, J., Metsch, P., Kästner, M., Borin, D. Y., Linke, J. M., and Odenbach, S., 2017. "Modeling of magnetic hystereses in soft mres filled with ndfeb particles". Smart Materials and Structures, 26(10), p. 105019.

[19] Kim, Y., Yuk, H., Zhao, R., Chester, S. A., and Zhao, X., 2018. "Printing ferromagnetic domains for untethered fast-transforming soft materials". Nature, 558(7709), pp. 274-279.

[20] Zhao, R., Kim, Y., Chester, S. A., Sharma, P., and Zhao, X., 2019. "Mechanics of hard-magnetic soft materials". Journal of the Mechanics and Physics of Solids, 124, pp. 244-263.

[21] Psarra, E., Bodelot, L., and Danas, K., 2019. "Wrinkling to crinkling transitions and curvature localization in a magnetoelastic film bonded to a non-magnetic substrate". Journal of the Mechanics and Physics of Solids, 133, p. 103734.

[22] Karami Mohammadi, N., Galich, P. I., Krushynska, A. O., and Rudykh, S., 2019. "Soft magnetoactive lam- inates: large deformations, transverse elastic waves and band gaps tunability by a magnetic field". Journal of Applied Mechanics, 86(11).

[23] Mukherjee, D., Bodelot, L., and Danas, K., 2020. "Microstructurally-guided explicit continuum models for isotropic magnetorheological elastomers with iron particles". International Journal of Non-Linear Mechanics, 120, p. 103380.

[24] Lefèvre, V., Danas, K., and Lopez-Pamies, O., 2020. "Two families of explicit models constructed from a homogenization solution for the magnetoelastic response of MREs containing iron and ferrofluid particles". International Journal of Non-Linear Mechanics, 119, p. 103362.

[25] Rambausek, M., and Danas, K., 2021. "Bifurcation of magnetorheological film-substrate elastomers subjected to biaxial pre-compression and transverse magnetic fields". International Journal of Non-Linear Mechanics, 128, p. 103608.

[26] Chen, W., and Wang, L., 2020. "Theoretical modeling and exact solution for extreme bending deformation of hard-magnetic soft beams". Journal of Applied Mechanics, 87(4).

[27] Zhang, R., Wu, S., Ze, Q., and Zhao, R., 2020. "Micromechanics study on actuation efficiency of hardmagnetic soft active materials". Journal of Applied Mechanics, 87(9).

[28] Schubert, G., and Harrison, P., 2015. "Large-strain behaviour of magneto-rheological elastomers tested under uniaxial compression and tension, and pure shear deformations". Polymer Testing, 42, pp. 122-134.

[29] Psarra, E., Bodelot, L., and Danas, K., 2017. "Twofield surface pattern control via marginally stable magnetorheological elastomers". Soft Matter, 13(37), pp. 6576-6584.

[30] Bodelot, L., Voropaieff, J.-P., and Pössinger, T., 2018. "Experimental investigation of the coupled magneto-mechanical response in magnetorheological elastomers". Experimental Mechanics, 58(2), pp. 207221.

[31] Pössinger, T., Bolzmacher, C., Bodelot, L., and Triantafyllidis, N., 2014. "Influence of interfacial adhesion on the mechanical response of magnetorheological elastomers at high strain". Microsystem Technologies, 20(4), pp. 803-814.

[32] Sutton, M. A., Orteu, J.-J., and Schreier, H. W., 2009. Image Correlation for Shape, Motion and Deformation Measurements. Basic Concepts, Theory and Applications. Springer, New York.

[33] Varga, Z., Filipcsei, G., and Zrínyi, M., 2006. "Magnetic field sensitive functional elastomers with tuneable elastic modulus". Polymer, 47(1), pp. 227 - 233.

[34] Danas, K., and Triantafyllidis, N., 2014. "Instability of a magnetoelastic layer resting on a non-magnetic substrate". Journal of the Mechanics and Physics of Solids, 69, pp. 67-83.

[35] Dorfmann, A., and Ogden, R., 2003. "Magnetoelastic modelling of elastomers". European Journal of 
Mechanics-A/Solids, 22(4), pp. 497-507.

[36] Thomas, J. D., and Triantafyllidis, N., 2009. "On electromagnetic forming processes in finitely strained solids: Theory and examples". Journal of the Mechanics and Physics of Solids, 57(8), pp. 1391-1416.

[37] Ammari, H., Buffa, A., and Nédélec, J.-C., 2000. "A justification of eddy currents model for the maxwell equations". SIAM Journal on Applied Mathematics, 60(5), pp. 1805-1823.

[38] Nédélec, J.-C., 1980. "Mixed finite elements in $\mathrm{r}^{3}$ ". Numerische Mathematik, 35, pp. 315-341.

[39] Coulomb, J.-L., 1981. "Finite elements three dimensional magnetic field computation". IEEE Transactions on Magnetics, 17(6), pp. 3241-3246.

[40] Biro, O., and Preis, K., 1989. "On the use of the magnetic vector potential in the finite-element analysis of three-dimensional eddy currents". IEEE Transactions on Magnetics, 25(4), pp. 3145-3159.

[41] Landis, C. M., 2002. "A new finite-element formulation for electromechanical boundary value problems". International Journal for Numerical Methods in Engineering, 55(5), pp. 613-628.

[42] Semenov, A. S., Kessler, H., Liskowsky, A., and Balke, H., 2006. "On a vector potential formulation for $3 \mathrm{~d}$ electromechanical finite element analysis". Communications in Numerical Methods in Engineering, 22(5), pp. 357-375.

[43] Stark, S., Semenov, A. S., and Balke, H., 2015. "On the boundary conditions for the vector potential formulation in electrostatics". International Journal for $\mathrm{Nu}$ merical Methods in Engineering, 102(11), pp. 17041732.

[44] Kankanala, S., and Triantafyllidis, N., 2004. "On finitely strained magnetorheological elastomers". Journal of the Mechanics and Physics of Solids, 52(12), pp. 2869-2908.

[45] Bustamante, R., Dorfmann, A., and Ogden, R. W., 2008. "On variational formulations in nonlinear magnetoelastostatics". Mathematics and Mechanics of Solids, 13(725-745).

[46] Lopez-Pamies, O., Goudarzi, T., and Danas, K., 2013. "The nonlinear elastic response of suspensions of rigid inclusions in rubber: II-a simple explicit approximation for finite-concentration suspensions". Journal of the Mechanics and Physics of Solids, 61(1), pp. 19-37.

[47] Forbes, L. K., Crozier, S., and Doddrell, D. M., 1997. "Rapid computation of static fields produced by thick circular solenoids". IEEE Transactions on Magnetics, 33(5), pp. 4405-4410. 